\title{
2017 Manchester Arena Bombing \\ Otological injuries and the need for a standard operating procedure for patients with acute hearing loss following blast injury
}

Herman A, Kearney DD, O’Driscoll M, Lloyd SK

\section{BACKGROUND}

- Otological injuries from primary blast exposures mainly manifest as hearing loss and tympanic membrane perforations. ${ }^{1}$

- Whilst acoustic injuries are not life threatening, their impact on the victim's activities of daily living can be significant; studies have shown that prompt identification of acute hearing loss allow for early administration of treatment such as glucocorticoids leading to better outcomes.

- On 22 May 2017, an improvised shrapnel bomb was detonated in the foyer area of Manchester Arena following a musical performance. The terrorist attack resulted in 22 deaths and more than eight hundred injured.

\section{OBJECTIVES AND METHODS}

- This study aims to report the otological injuries sustained by adult surviving victims of the attack who presented to Manchester Royal Infirmary (MRI) both at initial presentation and at follow up.

Further, we examine the hospital protocol used following the incident and suggestions for future iterations.

- A total of 21 adult patients underwent an initial standardised audiology assessment, mainly using portable equipment to allow bedside testing. Patients presenting to other hospitals or in the community were not included.

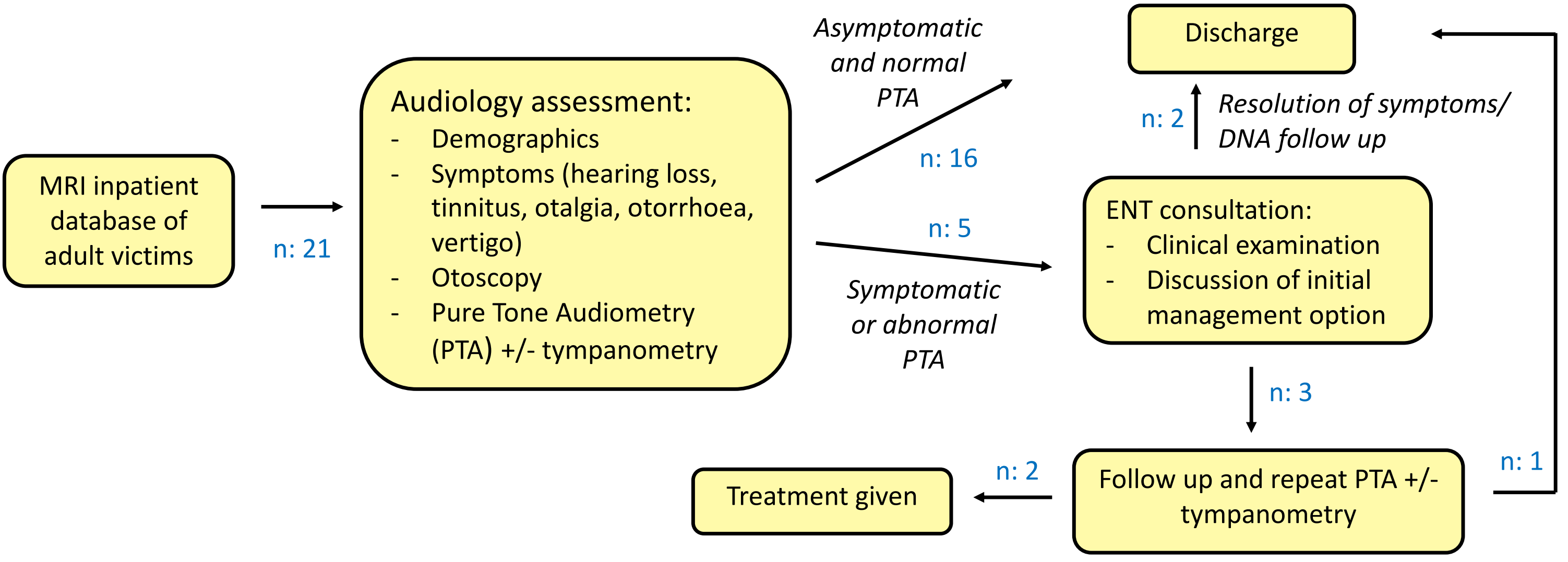

\section{RESULTS}

\section{Assessment Outcomes}

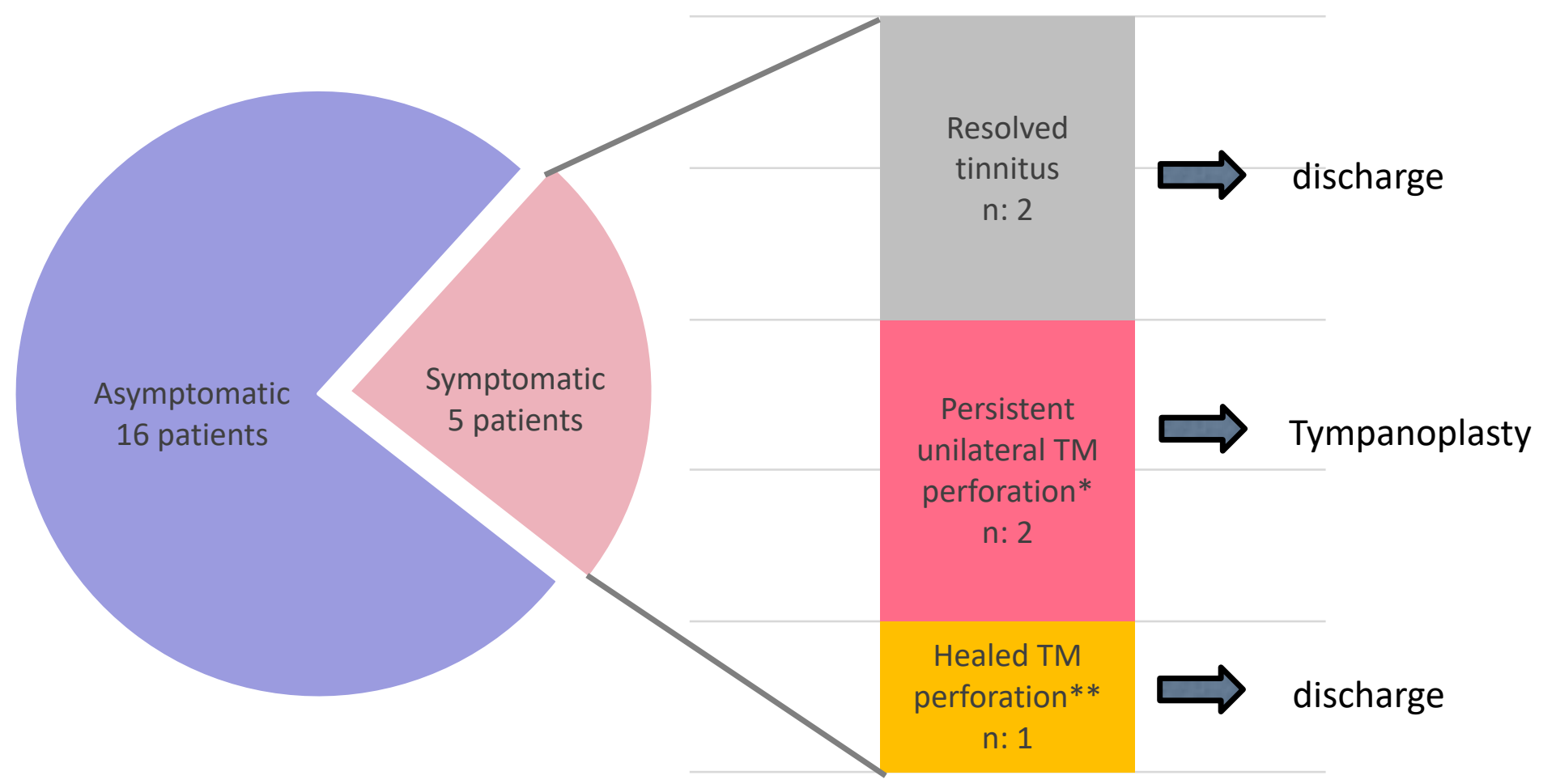

* CHL. No significant improvement at follow up on PTA and symptomatically

${ }^{* *} \mathrm{CHL}$. Resolution of symptoms with normal PTA and examination at follow up
First audiology assessment following incident

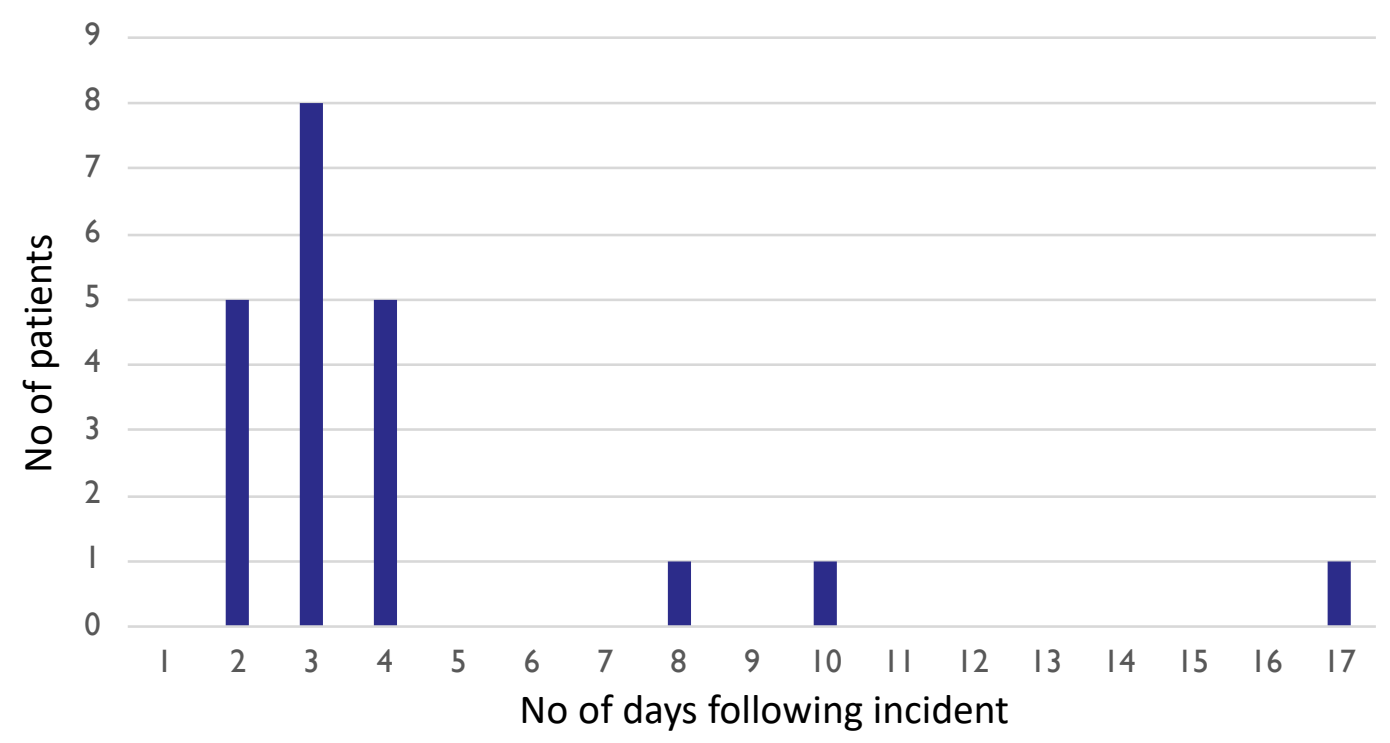

Factors affecting delay in first audiology encounter: At least 24 hour delay in information transfer from database to audiology department Limited portable audiometry equipment Critically ill patients

\section{CONCLUSION}

- Otological injuries represent significant morbidity following bombings. Whilst life and limb threatening injuries are correctly prioritised, it is important not to overlook the effects of otological injuries.

- Five cases were described in this study; three with conductive hearing loss and no sensorineural hearing loss recorded. However, it is likely that the total burden of otological trauma is significantly higher. The surprisingly low number of otological injuries may represent the distant location of surviving victims from the blast which provide a protective effect in dissipating the pressure wave.

It is likely that those with more significant acoustic injuries were closer to the blast and did not survive the incident.

- This supports the need for a standard operating procedure to aid early identification of victims. 